team, which is sent from the nuclear emergency medical support center, are unification and adjustment of the team activities from other facilities. Some other duties include offering appropriate medical care to patients at the disaster area and support of patients' transportation from the hospital at the disaster area to "advanced radiation emergency medical support center" or "nuclear emergency medical support center."

Discussion: In training the many facilities that participated, we realized that we couldn't proceed with each activity quickly and smoothly without support from the nuclear emergency medical assistance teams from outside the boundaries of disaster areas. We need to clarify the problems that are obtained from trainings and improve the current system corresponding to a nuclear disaster with efficiency.

Prehosp Disaster Med 2019;34(Suppl. 1):s170-s171

doi:10.1017/S1049023X1900390X

\section{A Systematic Review of Earthquake-Related Head Injuries} Dr. Yutaka Igarashi ${ }^{1}$, Ms Narumi Matsumoto ${ }^{2}$,

Dr. Tatsubiko Kubo ${ }^{3}$, Dr. Ryuta Nakae ${ }^{1}$, Dr. Shoji Yokobori ${ }^{1}$, Professor Hiroyuki Yokota ${ }^{1}$

1. Department of Emergency and Critical Care Medicine, Nippon Medical School Hospital, Tokyo, Japan

2. Faculty of Medicine, Nippon Medical School, Tokyo, Japan

3. Department of Environmental Epidemiology, Institute of Industrial Ecological Sciences, University of Occupational and Environmental Health, Kita-kyushu, Japan

Introduction: Earthquakes have killed around 800,000 people globally in the past 20 years, with head injury being the main cause of mortality and morbidity.

Aim: To conduct a systematic review to determine the characteristics of head injuries after earthquakes for better disaster preparedness and management.

Methods: All publications related to head injuries and earthquakes were searched using Pubmed, Web of Science, the Cochrane Library, and Ichushi.

Results: Thirty-six articles were included in the analysis. Head injury was the third most common cause of injury among survivors of earthquakes. The most common injury after an earthquake occurred was in the lower extremities (36.2\%), followed by the upper extremities (19.9\%), head (16.6\%), spine (13.3\%), chest (11.3\%), and abdomen (3.8\%). Earthquake-related head injuries were predominantly caused by a blunt strike (79\%), and were more frequently associated with soft tissue injury compared to non-earthquake-related head injuries and less frequently with intracranial hemorrhage. The mean age of patients with earthquake-related head injuries was 32.6 years, and $55.1 \%$ of sufferers were male. The most common earthquake-related head injury was laceration or contusion (59.2\%) while epidural hematoma was most common among inpatients with intracranial hemorrhage after an earthquake (9.5\%). Early wound irrigation and debridement and antibiotics administration are needed to decrease the risk of infection. Mortality due to earthquake-related head injuries was $5.6 \%$.
Discussion: Head injury was the main cause of mortality and morbidity after an earthquake. The characteristics of earthquake-related head injuries differed from those of non-earthquake-related head injuries, including the frequency of multiple injuries, and occurrence of contaminated soft tissue injury and epidural hematoma. This knowledge is important for determining demands for neurosurgery and for adequate management of patients, especially in resource-limited conditions.

Prehosp Disaster Med 2019;34(Suppl. 1):s171

doi:10.1017/S1049023X19003911

\section{Tabletop Simulation Exercise of Critically Ill Patient} Evacuation from a Hospital Fire

Ms Jiyoung Noh', Professor Hyun Soo Chung ${ }^{2}$, Ms Hye Mi Jin ${ }^{1}$, Ms Jayoung Hur ${ }^{1}$, Ms Min Ji Kim ${ }^{1}$, Ms Chan Mi Kang ${ }^{1}$,

Ms Ga Hyun Lee

1. Center for Disaster Relief, Training, and Research, Yonsei University Severance Hospital, Seoul, South Korea

2. Department of Emergency Medicine, Yonsei University College of Medicine, Seoul, South Korea

Introduction: Recent hospital fire incidents in South Korea have heightened the importance of patient evacuation. Moving patients from an intensive care unit (ICU) or emergency department (ED) setting is a challenge due to the complexity of moving acutely unwell patients who are reliant on invasive monitoring and organ support. Despite the importance of patient evacuation, the readiness of ICU and ED for urgent evacuation has not been assessed.

Aim: To enhance the readiness and competencies of workers from ICU and ED in the evacuation of patients during a simulated tabletop fire exercise.

Methods: A tabletop simulation exercise was developed by the Center for Disaster Relief, Training, and Research referencing the fire evacuation manual developed by the hospital's ICU and ED. The scenario consisted of evacuating patients horizontally and vertically from each department. The participants' actions were assessed using a checklist. A debriefing was completed after the exercise to discuss the gaps observed. A post-survey questionnaire was used to evaluate the exercise and assess the perception changes of the participants. All pre-to-post differences within subjects were analyzed with paired t-tests.

Results: A total of 22 and 29 people participated in the exercise from ICU and ED, respectively. Knowledge and confidence improved post-exercise for both ICU and ED scenarios $(p<0.05)$. Course satisfaction was 7.9 and 8.7, respectively for ICU and ED exercise. Correct performance rates for ICU and $\mathrm{ED}$ were $59 \%$ and 58\%, respectively. Common gaps noted for both ICU and ED were wearing protective masks, patient hand-over communication, and preparation for resources.

Discussion: There need to be exercises to recognize system gaps in place for hospital fire evacuation preparedness. Tabletop simulation exercises are ideal tools for this purpose. Although this was a short 90-minute exercise, this increased familiarity with the evacuation plan, tested the plan, and allowed for identification of gaps.

Prehosp Disaster Med 2019;34(Suppl. 1):s171

doi:10.1017/S1049023X19003923 